\title{
The Health and Social Care Bill: why all English people should be worried
}

Paul Batchelor

Consultant in Dental Public Health, Thames Valley Public Health Network

At the time of writing, the Health and Social Care Bill is continuing along its lengthy path towards assent. The current timetable envisages a series of debates in the upper chamber with the final reading in the House of Commons by the end of March and subsequent passage into law. The Secretary of State's inability (or unwillingness) to provide clarity on the precise rationale for the new legislation should raise concerns from all sectors of the health system as to the true reasons behind the new legislation. Only now are health professionals and the general public becoming fully aware of its likely consequences, previously misled by the reassuring words of a White Paper ${ }^{1}$ which purports to explain the content of the Bill while in fact disguising it: a velvet glove over an iron fist.

To date the limited comments on the Bill within the dental sector have addressed only its perceived impacts on dentists. Such an approach is narrow in thinking and fails to recognise the wider role of the dental professions, namely as advocates for patients with an appreciation of the wider determinants of oral health and how the care system can influence them. The Bill proposes major changes to the current delivery system, a separation of public health from health delivery and the use of competition as a mechanism to address the problems. This piece aims to summarise for readers what this Bill will mean for patients and their families.

In short, the Bill sets up a legal framework to enable the NHS to be changed permanently to the sort of health service that the USA and South Africa have, based around a commercial healthcare market. This change is being promoted in the face of extreme resistance, even though both the USA and South Africa have made considerable recent efforts to ditch their malfunctioning, expensive systems in favour of one more resembling the NHS. Many hundreds of amendments have been made to the Bill, but unfortunately none of them will have the effect of stopping this change, because the government controls the majority of votes in both Houses, and it will use the Whip to ensure that no meaningful amendment will pass. The amendments that have passed are helpful in persuading different constituencies that they have been listened to, and thus to defuse opposition and increase the chance that the Bill will pass. To use an analogy, imagine the Health and Social Care Bill as a car having parts amended. All of the amendments affect only the bodywork and upholstery. None of the changes have been made which might affect the engine, the wheels, the controls or other essential mechanisms.

\section{NHS IS TOP PERFORMER}

The National Health System is remarkably cost efficient. Studies comparing the systems of different developed countries show it performing consistently in the top few on both outcomes and affordability. All the rhetoric about NHS managerial bureaucracy and costs has minimal basis in reality, but appears intended to soften the public attitude to changes that will be unpopular once they bite. In contrast, the highly commercialised US healthcare industry on which the reform is modelled costs twice as much per person as the NHS, excludes much of the population from adequate care, and causes considerable morbidity and mortality through supplier-induced demand. There, 62\% of all bankruptcies are related to medical bills, and 78\% of those made bankrupt had valid medical insurance cover, with the typical family affected having a disabled or seriously ill child. ${ }^{2}$

The NHS is a mutual support system that protects every one of us from the possibility of ruinous medical bills. It is one of the elements that has shaped our society; it is one of the glues that unite us. Lose it and we become fragmented. England will be a much less pleasant place to live after the transformation of the NHS: a transformation from a comprehensive healthcare service to the 'great business' which David Cameron says is his vision of its future.

It is not just the delivery of dentistry that matters but how the public view the dental professions. The public's views of the professions will hardly be improved. The Bill fails to provide any logical argument on what may be tangible solutions to the problems that all delivery systems face. Look no further than primary dental care and how it has evolved, in particular its relationship with the NHS over the last decade. A contented profession? Improved relationships between the profession and the public? Less bureaucracy? While there is a need to debate how the NHS deals with the future demands that are expected to arise, this must be the starting point. Only then can any proposals be judged as to whether they are suitable. The dental profession would be wise to ask itself how the NHS reforms to date have impacted on it; reforms that the Bill will impose on the general healthcare system. It needs to examine the implications not just for dental care but for society as a whole.

p.batchelor@aul.ac.uk

1. Department of Health. Equity and excellence: liberating the NHS. London: Department of Health, 2010.

2. Woolhandler $\mathrm{S}$, Himmelstein D. Competition in a publicly funded healthcare system. BMJ 2007; 335: 1126-1129. 